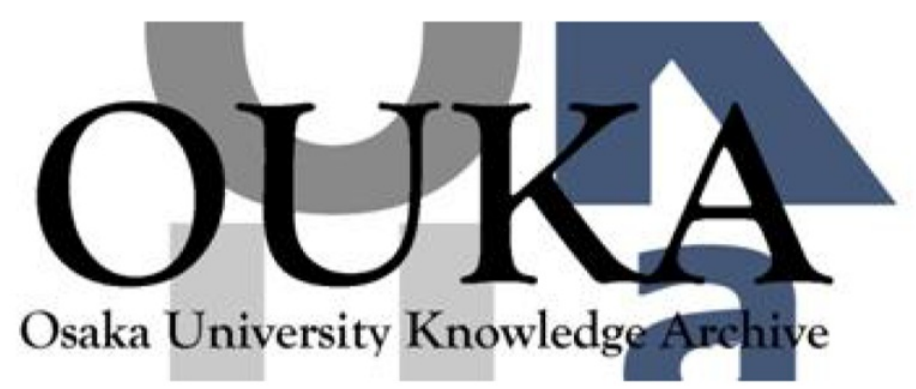

\begin{tabular}{|c|c|}
\hline Title & $\begin{array}{l}\text { Synthesis and solution properties of a rigid } \\
\text { helical star polymer: Three-Arm Star } \\
\text { Poly(quinoxaline-2,3-diyl) }\end{array}$ \\
\hline Author(s) & $\begin{array}{l}\text { Hasegawa, Hirokazu; Nagata, Yuuya; Terao, Ken } \\
\text { et al. }\end{array}$ \\
\hline Citation & Macromolecules. 50(19) p.7491-p. 7497 \\
\hline Issue Date & 2017-09-28 \\
\hline oaire:version & AM \\
\hline URL & https://hdl. handle. net/11094/81801 \\
\hline rights & $\begin{array}{l}\text { This document is the Accepted Manuscript } \\
\text { version of a Published Work that appeared in } \\
\text { final form in Macromolecules, @ American } \\
\text { Chemical Society after peer review and } \\
\text { technical editing by the publisher. To access } \\
\text { the final edited and published work see } \\
\text { https://doi.org/10.1021/acs. macromol. } 7 b 01797 \text {. }\end{array}$ \\
\hline Note & \\
\hline
\end{tabular}

Osaka University Knowledge Archive : OUKA

https://ir. Library. osaka-u. ac. jp/

Osaka University 


\section{Synthesis and Solution Properties of a Rigid Helical Star Polymer: Three-arm Star Poly(quinoxaline-2,3- diyl)}

Hirokazu Hasegawa, ${ }^{\dagger+}$ Yuuya Nagata, ${ }^{*}$, Ken Terao,,$+\dagger$ and Michinori Suginome ${ }^{\S}$

†Department of Macromolecular Science, Graduate School of Science, Osaka University, 1-1 Machikaneyama-cho, Toyonaka, Osaka 560-0043, Japan

${ }^{\sharp}$ Materials Characterization Laboratories, Toray Research Center, Inc., 3-3-7, Sonoyama, Otsu, Shiga 520-8567, Japan

${ }^{\S}$ Department of Synthetic Chemistry and Biological Chemistry, Graduate School of Engineering, Kyoto University, Katsura, Kyoto 615-8510, Japan

Corresponding Authors

*E-mail: nagata@sbchem.kyoto-u.ac.jp

*E-mail:kterao@chem.sci.osaka-u.ac.jp

ABSTRACT: Six semiflexible helical three-arm star poly[5,8-dimethyl-6,7bis(propoxymethyl)quinoxaline-2,3-diyl] (A3PQ) samples which have different arm length ranging in the weight-average molar mass $M_{\mathrm{w}}$ from $1.5 \times 10^{4} \mathrm{~g} \mathrm{~mol}^{-1}$ to $2.5 \times 10^{5} \mathrm{~g} \mathrm{~mol}^{-1}$ were synthesized via the living polymerization of the corresponding 1,2-diisocyanobenzene using a trifunctional palladium initiator. The particle scattering function $P(q)$, the $z$-average meansquare radius of gyration $\left\langle S^{2}\right\rangle_{z}$, and the intrinsic viscosity $[\eta]$ for the A3PQ samples were determined from light and small-angle $\mathrm{X}$-ray scattering and viscosity measurements in tetrahydrofuran at $25{ }^{\circ} \mathrm{C}$. The obtained data were compared with modern theories for the wormlike star. The experimental $P(q)$ data were represented by the wormlike star model connected with a universal joint. The other data were comparably explained by the same model.

\section{Introduction}

Star polymers are a class of simple non-linear polymers. Well-defined star polymers consisting of flexible arms are thoroughly investigated both theoretically and experimentally. ${ }^{1-2}$ Conformational properties of stiff polymer chains are more readily to be understood theoretically since intramolecular excluded-volume effects are much less important than those for the flexible polymer chains. ${ }^{3}$ Experimental researches to determine gyration radii and/or intrinsic viscosities of semiflexible or rigid star polymers are however limited to poly( $\gamma$-benzyl-L-glutamate $){ }^{4}$ poly $(n$-hexylisocyanate $),{ }^{5-6}$ and specially designed 'polypod' DNA. ${ }^{7}$ Polymer conformations of such star polymers are important to understand their structure-function relationships if we consider some star polymers with rigid (helical) arms are considerably attractive because of their unique features, that is, molecular recognition ${ }^{8}$ and self-assembly behavior. ${ }^{9-11}$ Quantitative discussion of dimensional and hydrodynamic properties in terms of the modern theories ${ }^{12}$ are however limited. Taking into account that the heterogeneity of the main chain and relatively large core of the star polymers significantly influence their dimensional properties as seen in a triple helical peptide with a linker domain, ${ }^{13}$ star polymers consisting of rigid homopolymer and well defined core are indispensable to clarify conformational features of semiflexible stars. 
We thus synthesized a three-arm star polymer (A3PQ) having rigid helical poly[5,8dimethyl-6,7-bis(propoxymethyl)quinoxaline-2,3-diyl] (PQX) with a well-designed threefunctional initiator. A linear PQX behaves as a semi-rigid polymer of which Kuhn segment length $\left(\lambda^{-1}\right)$ was reported to be $43 \mathrm{~nm}$ in tetrahydrofuran (THF) at $25{ }^{\circ} \mathrm{C} .{ }^{14}$ It is thus a candidate as a unique class of functional polymers because linear poly(quinoxaline-2,3-diyl)s are useful as highly effective chiral ligands for transition metal catalysts in asymmetric reactions, ${ }^{15}$ and furthermore, they may have solvent dependent helix reversal behaviors. ${ }^{16-17}$ We prepared A3PQ samples with different molar mass. Light scattering, small-angle X-ray scattering, and viscosity measurements were made for the samples in THF to determine their dimensional and hydrodynamic properties. The resultant solution data were analyzed in terms of the modern theories for wormlike stars to clarify their molecular conformation in solution.

\section{Experimental Section}

Synthesis of A3PQ. Six A3PQ samples were synthesized by the core first method as shown in Schemes 1 and 2. All reactions were carried out under a nitrogen atmosphere. 1,3,5Tris-(4-iodo-phenyl)-benzene (1) ${ }^{18} \quad$ 1,2-diisocyano-3,6-di- $p$-tolylbenzene $\quad(\mathbf{3}),{ }^{19}$ and $1,2-$ diisocyano-3,6-dimethyl-4,5-bis(propoxymethyl)benzene $(\mathbf{5})^{20}$ were prepared according to the reported procedures. Other chemical reagents were purchased from the commercial sources and were used without further purification.

Scheme 1. Synthesis of the trifunctional palladium initiator 4.
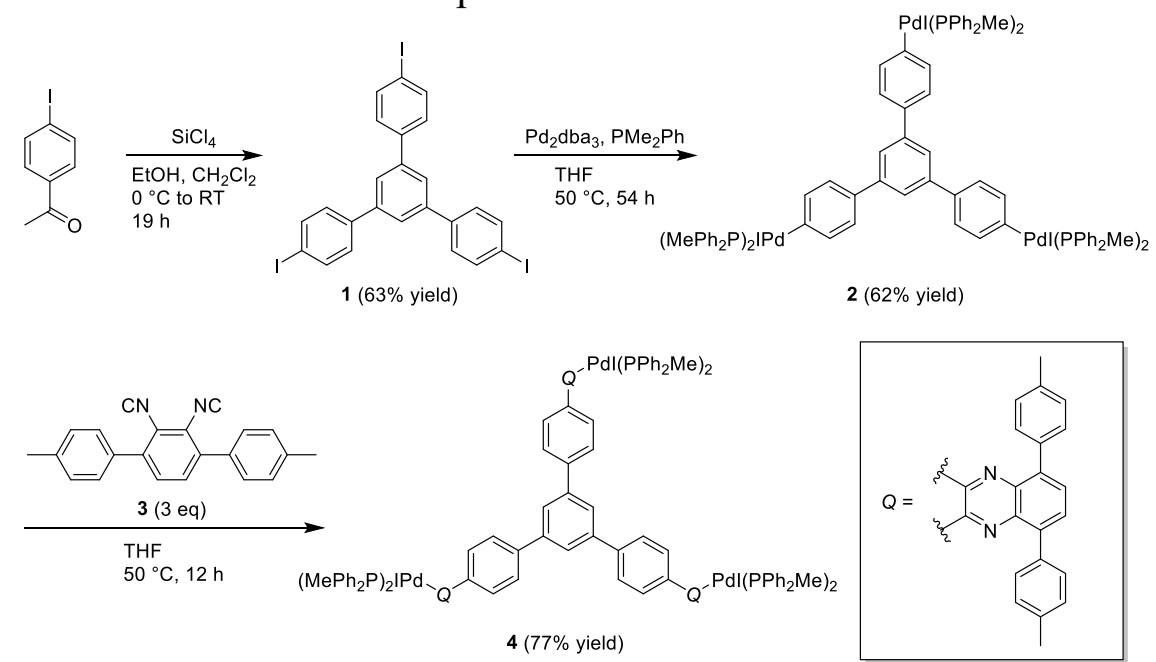

Scheme 2. Polymerization of 1,2-dicyanobenzene monomer 5 with the trifunctional palladium initiator 4. 


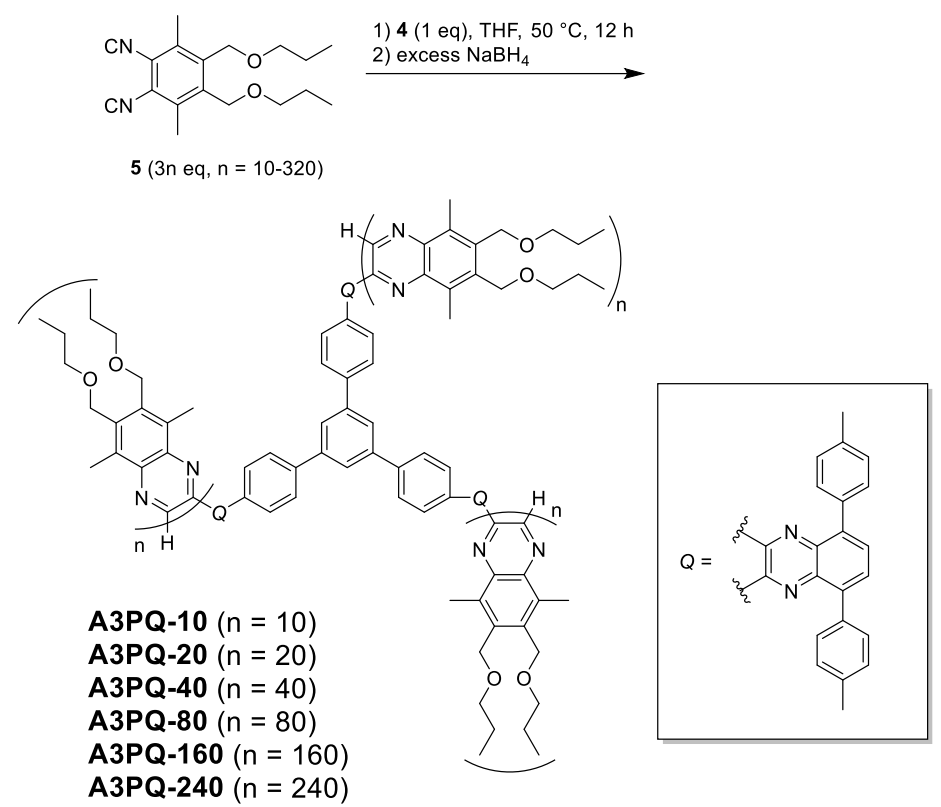

A procedure to synthesize a trifunctional palladium initiator $\mathbf{4}$ is as follow: Tris(dibenzylideneacetone)dipalladium $(0) \quad\left(\mathrm{Pd}_{2} \mathrm{dba}_{3}, \quad 1.30 \quad \mathrm{~g}, \quad 1.42 \quad \mathrm{mmol}\right)$ and dimethylphenylphosphine ( $\left.\mathrm{PMe}_{2} \mathrm{Ph}, 0.82 \mathrm{~mL}, 5.79 \mathrm{mmol}\right)$ were dissolved in THF $(5.85 \mathrm{~mL})$ and stirred for $10 \mathrm{~min}$. A THF solution of $\mathbf{1}(0.4 \mathrm{~g}, 0.584 \mathrm{mmol}$ in $11.69 \mathrm{~mL}$ of THF) was added to the reaction mixture and stirred at $50{ }^{\circ} \mathrm{C}$ for $54 \mathrm{~h}$. After the reaction, the reaction mixture was filtered through a pad of Celite and washed with dichloromethane $\left(\mathrm{CH}_{2} \mathrm{Cl}_{2}, 50 \mathrm{~mL}\right)$. The filtrate was concentrated under reduced pressure. The residual brown solid was subjected to a silica gel column chromatography using $\mathrm{CH}_{2} \mathrm{Cl}_{2}$ as an eluent $\left(R_{\mathrm{f}}=0.42\right.$ using $\left.\mathrm{CH}_{2} \mathrm{Cl}_{2}\right)$. After evaporation to dryness, the crude product was dissolved in $\mathrm{CH}_{2} \mathrm{Cl}_{2}(5 \mathrm{~mL})$ and reprecipitated into methanol $(50 \mathrm{~mL})$. The precipitate was washed with methanol $(50 \mathrm{~mL})$ and dried under vacuum to give white solid of $2(0.66 \mathrm{~g}, 62 \%$ yield $)$. Compounds $2(0.300 \mathrm{~g}, 0.164 \mathrm{mmol})$ and $\mathbf{3}(0.152 \mathrm{~g}, 0.491$ mmol) were dissolved in THF $(9.8 \mathrm{~mL})$. The reaction mixture was stirred at $50{ }^{\circ} \mathrm{C}$ for $24 \mathrm{~h}$. After evaporation of the reaction mixture to dryness, the residual solid was subjected to a silica gel column chromatography using $n$-hexane and $\mathrm{CH}_{2} \mathrm{Cl}_{2}$ ( $n$-hexane/ $\mathrm{CH}_{2} \mathrm{Cl}_{2}=25 / 75$ to $0 / 100, R_{\mathrm{f}}$ $=0.50$ using $n$-hexane/ $\left.\mathrm{CH}_{2} \mathrm{Cl}_{2}=25 / 75\right)$ to give 4 as a yellow solid $(0.35 \mathrm{~g}, 77 \%$ yield $)$.

A typical procedure for the polymerization is as follows: A3PQ-40: Compound 4 (0.0129 $\mathrm{mol} / \mathrm{L}, 0.71 \mathrm{~mL})$ was added to a solution of $5(0.1271 \mathrm{~g}, 0.4232 \mathrm{mmol})$ in THF $(16.9 \mathrm{~mL})$. The reaction mixture was stirred at $50{ }^{\circ} \mathrm{C}$. After $12 \mathrm{~h}$, excess amount of $\mathrm{NaBH}_{4}(0.0534 \mathrm{~g}, 1.41$ mmol) was added, and the reaction mixture was stirred for $2 \mathrm{~h}$ at room temperature. The resultant mixture was poured into water $(50 \mathrm{~mL})$ and extracted with chloroform $(50 \mathrm{~mL} \times 3)$. The organic extract was dried over $\mathrm{MgSO}_{4}$, and the solvent was evaporated. The residue was subjected to preparative size exclusion chromatography (SEC) to give A3PQ-40 as a brown solid (126.5 mg, 96\% yield). Six A3PQ samples, A3PQ-10, A3PQ-20, A3PQ-40, A3PQ-80, A3PQ160, and A3PQ-240 of which weight-average molar mass $M_{\mathrm{w}}$ ranges from $1.5 \times 10^{4} \mathrm{~g} \mathrm{~mol}^{-1}$ to $2.5 \times 10^{5} \mathrm{~g} \mathrm{~mol}^{-1}$ were eventually prepared for this study. The chemical structures of the resultant polymer and the initiator were confirmed by ${ }^{1} \mathrm{H}$ NMR and/or X-ray analysis as reported in the Supporting Information. 
Static Light Scattering (SLS) and Viscosity Measurements with SEC. SLS and viscosity measurements for all samples in THF were made using SEC equipped with a DAWN HELEOS multi-angle laser light scattering photometer, an Optilab rEX differential refractometer, and a VISCOSTAR-II differential viscometer (Wyatt Technology, USA) at room temperature $(\sim$ $25{ }^{\circ} \mathrm{C}$ ). Two TSKgel GMHXL columns and a G2500HxL column connected in series were used for the SEC system. Details of the experimental and analytical procedures were the same as those for the corresponding linear PQX as mentioned previously. ${ }^{14}$ Differential refractive index increments $\partial n / \partial c$ for all A3PQ samples in THF at the wavelength in vacuum $\left(\lambda_{0}\right)$ of the light scattering photometer were determined by using the peak area of the refractive index chromatogram and the polymer mass concentration $c$ of injected solution assuming full recovery of the A3PQ sample. The $\partial n / \partial c$ value was also determined for A3PQ-80 with an Optilab T-rEX differential refractometer (Wyatt) by a batch method to be $0.192 \mathrm{~cm}^{3} \mathrm{~g}^{-1}$, which is the same as that from the former method. The weight-average molar mass $M_{\mathrm{w}}$, the intrinsic viscosity $[\eta]$, and the $z$-average mean-square radius of gyration $\left\langle S^{2}\right\rangle_{z}$ for the A3PQ samples were determined at each retention volume $V_{\mathrm{e}}$. We note that the light scattering data were analyzed in terms of the Berry square-root plot $^{21}$ and the values of $\left\langle S^{2}\right\rangle_{z}$ were not determined for A3PQ-10, A3PQ-20, A3PQ-40, and A3PQ-80 because they were less than the lower limit $\left(\sim 100 \mathrm{~nm}^{2}\right)$ of the light scattering detector. The resultant $M_{\mathrm{w}}$ values determined from the peak area agreed with those from the universal calibration method ${ }^{22-23}$ within $\pm 3 \%$. The dispersity index $Ð$ defined as the ratio of $M_{\mathrm{w}}$ to the number average molar mass $M_{\mathrm{n}}$ from the two methods was also determined from the two methods. The SEC chromatograms for A3PQ-80, A3PQ-160, and A3PQ-240 have a small shoulder peak suggesting high molar mass component as displayed in Figure 1. Similar component was also observed for the linear PQX samples. ${ }^{14}$ The shoulder peaks were thus omitted in the following analyses.

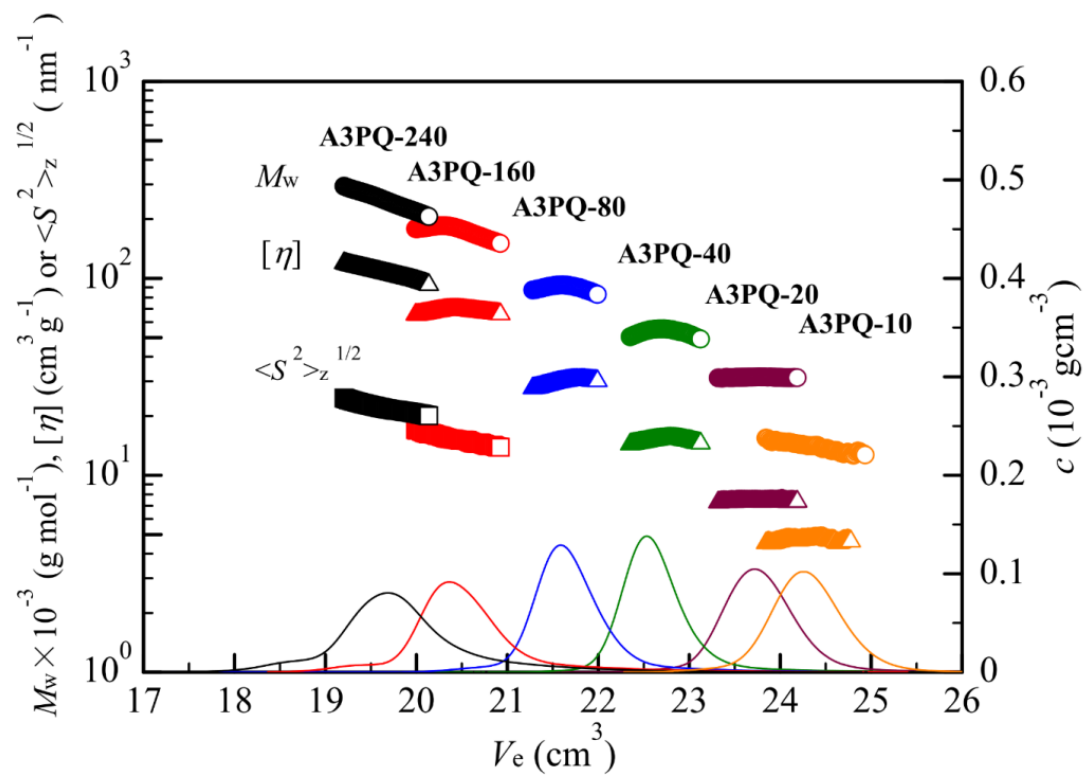

Figure 1. Retention volume $V_{\mathrm{e}}$ dependence of the weight-average molar mass $M_{\mathrm{w}}$ (circles), the $z$-average radius of gyration $\left\langle S^{2}\right\rangle_{z}^{1 / 2}$ (squares), the intrinsic viscosity [ $\left.\eta\right]$ (triangles), and the polymer mass concentration $c$ (solid curves) for the indicated A3PQ samples in THF at room temperature $\left(\sim 25^{\circ} \mathrm{C}\right)$. 
Small angle X-ray Scattering (SAXS). SAXS measurements were performed for A3PQ10, A3PQ-20, A3PQ-40, A3PQ-80, and A3PQ-160 in THF at $25{ }^{\circ} \mathrm{C}$ using a Rigaku R-AXIS VII imaging plate at the BL40B2 beamline in SPring-8 (Hyogo, Japan). The camera length, $\lambda_{0}$, and the accumulation time were set to be $4 \mathrm{~m}, 0.1 \mathrm{~nm}$, and $180 \mathrm{sec}$, respectively. The magnitude $q$ of the scattering vector at each pixel was determined from the diffraction pattern of silver behenate. The measurements were carried out for the solvent and four solutions having different $c$ ranging in $6 \times 10^{-3} \mathrm{~g} \mathrm{~cm}^{-3}$ and $3 \times 10^{-2} \mathrm{~g} \mathrm{~cm}^{-3}$. The scattering intensity $I(q)$ was obtained as a function of $q$ by the circular average procedure. The excess scattering intensity $\Delta I(q)$ of each solution was determined from the difference between $I(q)$ of the solution and that of the solvent in the same capillary cell taking the intensity and transparency of the incident X-ray into account. The $[c / \Delta I(q)]^{1 / 2}$ data were extrapolated to infinite dilution and to $q^{2}=0$ to estimate the particle scattering function $P(q)$. Figure 2 shows the Berry plot of the resultant $P(q)$ data to determine $\left\langle S^{2}\right\rangle_{\mathrm{z}}$. Since the extrapolation to $q^{2}=0$ was infeasible for A3PQ-160, we estimated the $P(q)$ to fit the experimental data at the lowest $q$ to the dashed line of which initial slope was drawn to represent the $\left\langle S^{2}\right\rangle_{Z}$ value determined from light scattering measurements.

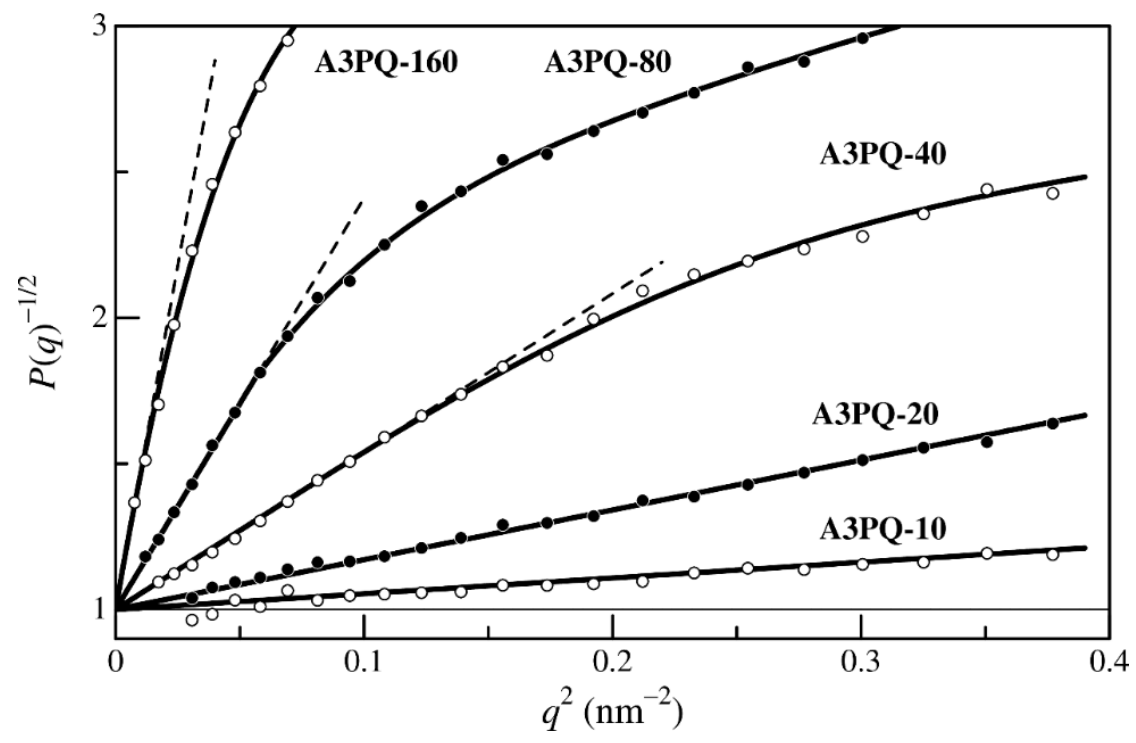

Figure 2. Berry square-root plots for indicated A3PQ samples in THF at $25{ }^{\circ} \mathrm{C}$. Dashed lines indicate the initial slopes.

\section{Results}

Table 1 summarizes numerical data for $\partial n / \partial c, M_{\mathrm{w}}, \oslash\left(\equiv M_{\mathrm{w}} / M_{\mathrm{n}}\right),\left\langle S^{2}\right\rangle_{\mathrm{z}}$, and [ $\left.\eta\right]$ mainly determined from the above mentioned SEC and SAXS measurements. The quite small $Ð$ values for the A3PQ samples suggest the relatively narrow arm length distribution while direct observation has not been examined. The $\partial n / \partial c$ values for the two lowest $M_{\mathrm{w}}$ samples, A3PQ-10 and A3PQ-20, are somewhat larger than those for the higher molar mass samples. This is most likely due to the higher refractive index of the core region. 
Table 1. Molecular Characteristics of Three-arm Star Poly[5,8-dimethyl-6,7bis(propoxymethyl)quinoxaline-2,3-diyl] (A3PQ) Samples and Physical Properties in THF at $25^{\circ} \mathrm{C}$ or Room Temperature $\left(25^{\circ} \mathrm{C}\right)$

\begin{tabular}{|c|c|c|c|c|c|c|c|}
\hline Sample & $\begin{array}{c}\partial n / \partial c \\
\left(\mathrm{~cm}^{3} \mathrm{~g}^{-1}\right)\end{array}$ & $\begin{array}{c}M_{\mathrm{W}} \\
\left(10^{-3} \mathrm{~g}\right. \\
\left.\mathrm{mol}^{-1}\right) \\
\end{array}$ & $\begin{array}{c}Ð \\
\left(M_{\mathrm{w}} / M_{\mathrm{n}}\right) \\
\end{array}$ & $\begin{array}{c}\left\langle S^{2}\right\rangle_{\mathrm{z}}^{1 / 2} \\
(\mathrm{~nm})\end{array}$ & $\begin{array}{c}{[\eta]} \\
\left(\mathrm{cm}^{3} \mathrm{~g}^{-1}\right)\end{array}$ & $g_{s}$ & $g_{\eta}$ \\
\hline A3PQ-10 & 0.207 & 14.8 & $\begin{array}{l}1.01^{\mathrm{b}} \\
1.09^{\mathrm{c}}\end{array}$ & $1.8^{\mathrm{a}}$ & 4.8 & 0.51 & 0.69 \\
\hline A3PQ-20 & 0.199 & 31.9 & $\begin{array}{l}1.01^{\mathrm{b}} \\
1.15^{\mathrm{c}}\end{array}$ & $3.2^{\mathrm{a}}$ & 7.3 & 0.38 & 0.47 \\
\hline A3PQ-40 & 0.190 & 52.9 & $\begin{array}{l}1.06^{\mathrm{b}} \\
1.18^{\mathrm{c}}\end{array}$ & $5.7^{\mathrm{a}}$ & 14.4 & 0.48 & 0.53 \\
\hline A3PQ-80 & 0.192 & 88.8 & $\begin{array}{l}1.04^{b} \\
1.08^{\mathrm{c}}\end{array}$ & $9.1^{\mathrm{a}}$ & 29.0 & 0.51 & 0.58 \\
\hline A3PQ-160 & 0.189 & 176 & $\begin{array}{l}1.07^{\mathrm{b}} \\
1.19^{\mathrm{c}}\end{array}$ & $16.8^{\mathrm{b}}$ & 67.0 & 0.60 & 0.62 \\
\hline A3PQ-240 & 0.187 & 245 & $\begin{array}{l}1.18^{b} \\
1.16^{\mathrm{c}}\end{array}$ & $22.0^{\mathrm{b}}$ & 103 & 0.65 & 0.67 \\
\hline
\end{tabular}

${ }^{a}$ From SAXS. ${ }^{b}$ From SEC-SLS. ${ }^{c}$ From universal calibration.

Molar mass dependence of the resultant $\left\langle S^{2}\right\rangle_{\mathrm{z}}{ }^{1 / 2}$ data for the A3PQ samples are illustrated in Figure 3 . They are systematically smaller than those for the corresponding linear polymer. The factor $g_{\mathrm{s}}$ defined as

$$
g_{\mathrm{s}}=\frac{\left\langle S^{2}\right\rangle_{\mathrm{star}}}{\left\langle S^{2}\right\rangle_{\text {linear }}}
$$

was calculated using the theoretical values for the corresponding linear chain. ${ }^{14}$ The resultant $g_{\mathrm{s}}$ values are listed in the 7th column in Table 1 . These values are between 1/3 and 7/9 for rod like and Gaussian three arm stars, respectively (see later). The Holtzer plot of $P(q)$ has an appreciable peak at low $q$ region and it becomes mostly flat with increasing $q$ as illustrated in Figure 4 while such peak was not seen in those for the corresponding linear chain. ${ }^{14}$ This is most likely due to the smaller dimensions of the star polymers than those for the corresponding linear chain with the same $M_{\mathrm{w}}$. 


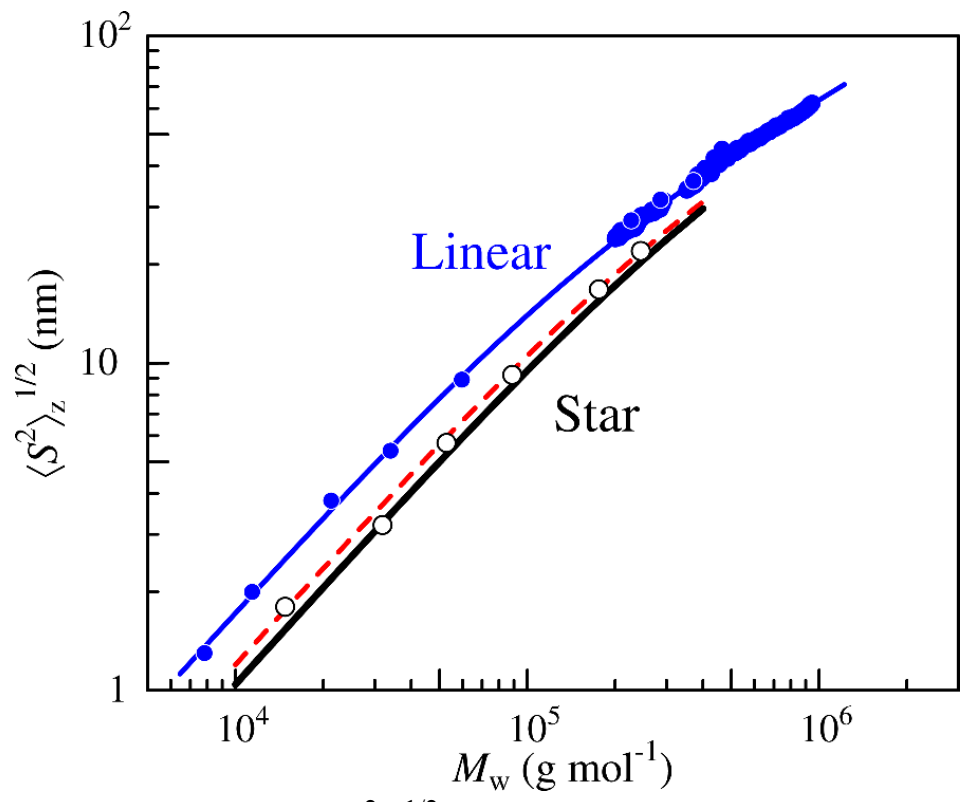

Figure 3. Double logarithmic plots of $\left\langle S^{2}\right\rangle_{\mathrm{z}}{ }^{1 / 2}$ vs $M_{\mathrm{w}}$ for A3PQ (unfilled circles) and PQX (filled circles) in THF at $25^{\circ} \mathrm{C}$ or the room temperature. Solid black and dashed red curves indicate the broken $\left(\left\langle S^{2}\right\rangle_{\mathrm{BW}}\right.$, eq 9) and fixed $\left(\left\langle S^{2}\right\rangle_{\mathrm{FIX}}\right.$, eq 10) wormlike chains, respectively, with the parameters shown in the text.

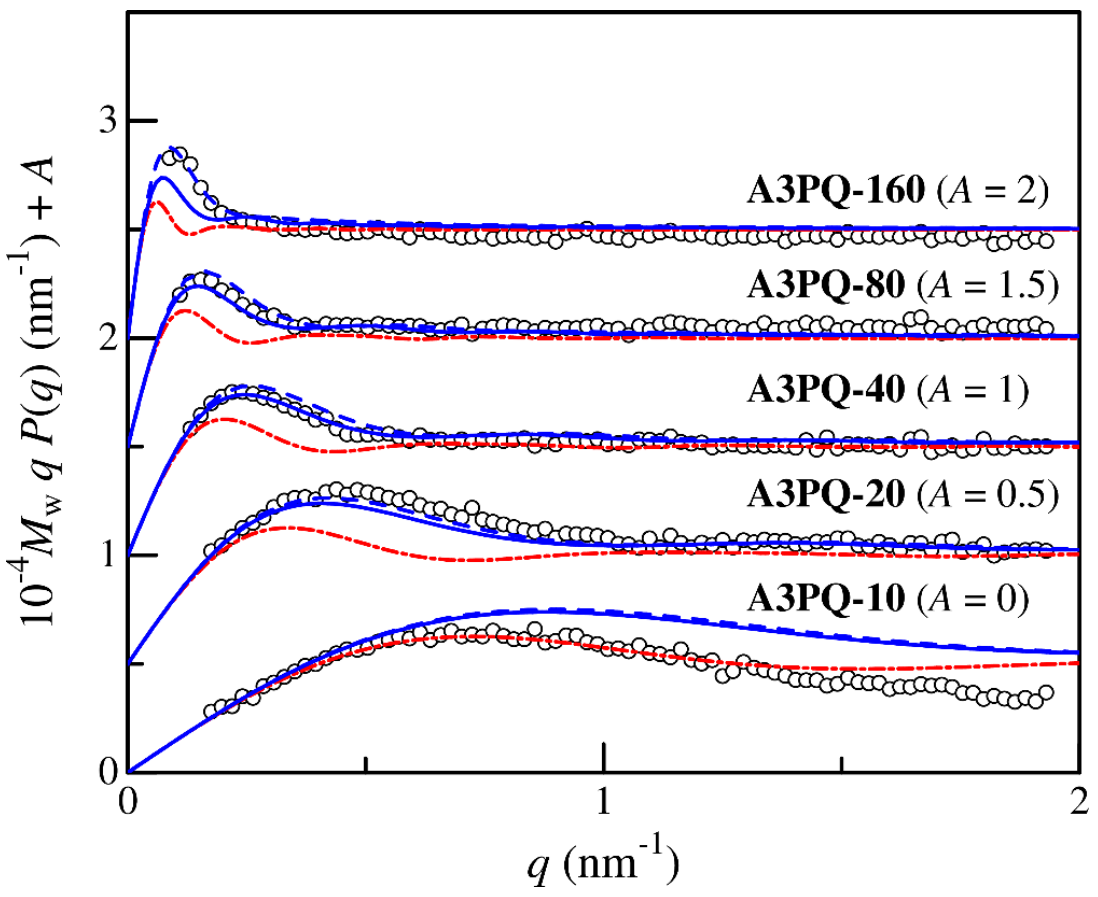

Figure 4. Reduced Holtzer plots for the indicated A3PQ samples in THF at $25{ }^{\circ} \mathrm{C}$. Solid and dashed blue curves denote the theoretical values for the rigid $\left[P_{\mathrm{BR}}(q)\right.$, eq 3] and wormlike $\left[P_{\mathrm{BW}}(q)\right.$, eq 8] broken star, respectively, with the parameters written in the text. The dot-dashed red curves represent the theoretical values of the fixed star $\left[P_{\mathrm{FIX}}(q)\right.$, eq 7]. The ordinate values are shifted by $A$ for clarity. 
Figure 5 shows molar mass dependence of $[\eta]$ for A3PQ samples in THF along with those for the corresponding linear chains. As in the case of $g_{\mathrm{s}}$, the $g_{\eta}$ factor defined as

$$
g_{\eta}=\frac{[\eta]_{\text {star }}}{[\eta]_{\text {linear }}}
$$

was calculated with the theoretical value for the corresponding linear chain and summarized in the 8th column in Table 1. As also displayed in Figure 5, the obtained $g_{\eta}$ values are between 4/9 and 0.90 for rigid $^{24}$ and flexible stars, ${ }^{25}$ respectively; note that the former value is calculated for rigid stars with the fixed connection angle. Except for the lowest $M_{\mathrm{w}}$ sample, the obtained $g_{\eta}$ values increase with increasing $M_{\mathrm{w}}$. These features for dimensional and hydrodynamic properties strongly suggest the semiflexible nature of the current star polymer samples.

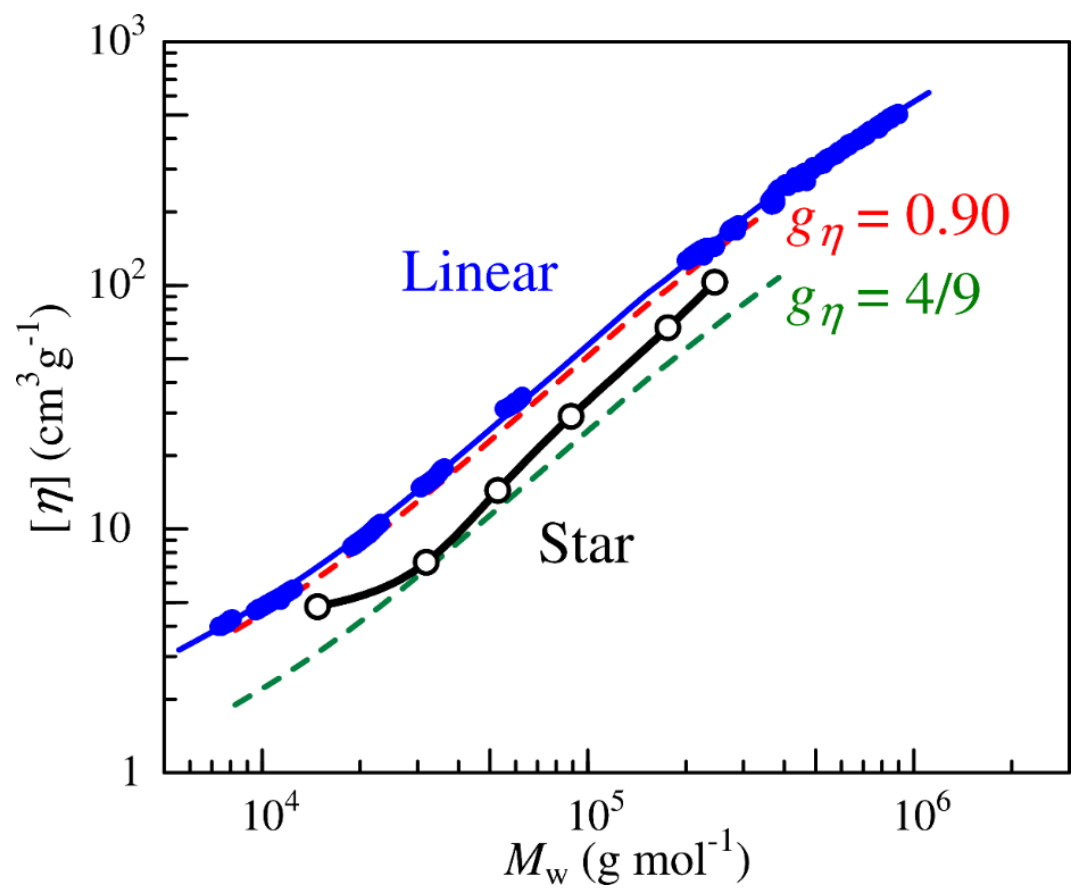

Figure 5. Double logarithmic plots of [ $\eta$ ] vs $M_{\mathrm{w}}$ for the A3PQ (unfilled circles) and PQX (filled circles) samples in $\mathrm{THF}$ at $25^{\circ} \mathrm{C}$. Dashed red and green curves represent the calculated values for $g_{\eta}=0.9$ and $4 / 9$, respectively.

\section{Discussion}

Wormlike Chain Analysis of $\boldsymbol{P}(\boldsymbol{q})$. Since both dimensional and hydrodynamic properties suggest the current star polymer behaves as rodlike or semiflexible stars, rod and wormlike star chain models are introduced to analyze the experimental data. According to our recent study, ${ }^{14}$ $P(q)$ and $\left\langle S^{2}\right\rangle_{\mathrm{z}}{ }^{1 / 2}$ data for the linear PQX were explained by the rigid rod model when $M_{\mathrm{w}}<3 \times$ $10^{4}$, suggesting that each arm of the star samples with the $M_{\mathrm{w}}$ value being less than $9 \times 10^{4}$ may be modeled by the rod star model. According to Huber and Burchard, ${ }^{26}$ the scattering function $P_{\mathrm{BR}}(q)$ of the broken three-arm rodlike star having uniform arm length $L_{\mathrm{s}}$ can be expressed as 


$$
P_{\mathrm{BR}}(q)=\frac{2}{3 q^{2} L_{\mathrm{S}}^{2}}\left\{q L_{\mathrm{S}} \mathrm{Si}\left(q L_{\mathrm{S}}\right)+\cos \left(q L_{\mathrm{S}}\right)-1+\left[\operatorname{Si}\left(q L_{\mathrm{S}}\right)\right]^{2}\right\}
$$

where $\operatorname{Si}(z)$ is the sine integral defined as

$$
\operatorname{Si}(z)=\int_{0}^{z} \frac{\sin t}{t} \mathrm{~d} t
$$

Each arm of this model is linked with a universal joint. The contour length $L_{\mathrm{s}}$ of each arm is one-third of the total contour length $L_{\mathrm{t}}$, which is proportional to $M_{\mathrm{w}}$. Thus, an $M_{\mathrm{w}}$ independent parameter $h$, helix pitch (or rise) per residue is defined as

$$
h=\frac{L_{\mathrm{t}} M_{0}}{M_{\mathrm{w}}}=\frac{3 L_{\mathrm{s}} M_{0}}{M_{\mathrm{w}}}
$$

where $M_{0}$ is the molar mass of the repeat unit $\left(=300.2 \mathrm{~g} \mathrm{~mol}^{-1}\right)$. The chain thickness may affect $P(q)$ in the high $q$ range. The touched bead model with the diameter $d$ is utilized to consider this effect. The resultant scattering function $P(q)$ is expressed as

$$
P(q)=9\left(\frac{2}{q d}\right)^{6}\left(\sin \frac{q d}{2}-\frac{q d}{2} \cos \frac{q d}{2}\right)^{2} P_{0}(q)
$$

where $P_{0}(q)$ is the particle scattering function of the thin chain; in this case, $P_{\mathrm{BR}}(q)$. If we assume previously estimated $h$ of $0.19 \mathrm{~nm}$ and $d=0.4 \mathrm{~nm}$, the solid blue curves which are the theoretical $P(q)$ values from eq 6 with eq 3 reproduce the experimental data almost quantitatively except for the lowest and highest $M_{\mathrm{w}}$ samples. It should be noted that the chain thickness effect on $P(q)$ was invisibly small in the current $q$ range. In order to consider the bond angle between the two arms, we also calculated the fixed rodlike star by

$$
P_{\mathrm{FIX}}(q)=\frac{2\left[q L_{\mathrm{S}} \mathrm{Si}\left(q L_{\mathrm{S}}\right)+\cos \left(q L_{\mathrm{S}}\right)-1\right]}{3 q^{2} L_{\mathrm{S}}^{2}}+\frac{2}{3 L_{\mathrm{S}}^{2}} \int_{0}^{L_{\mathrm{s}}} \mathrm{d} s_{0} \int_{0}^{L_{\mathrm{S}}} \mathrm{d} s_{1} \frac{\sin q \sqrt{s_{0}^{2}+s_{1}{ }^{2}+s_{0} s_{1}}}{q \sqrt{s_{0}^{2}+s_{1}^{2}+s_{0} s_{1}}}
$$

when we choose $120^{\circ}$ as the bond angle between arm chains. Dot-dashed red curves in Figure 4 represent the theoretical $P(q)$ for the fixed rodlike star calculated from eq 7. They clearly underestimate $P(q)$ around the peak except for the lowest $M_{\mathrm{w}}$ sample. The nicely fitted theoretical values for the lowest $M_{\mathrm{w}}$ sample may be accidental because the chain end effect as well as the core effect on $P(q)$ should be more significant for the sample. Taking into consideration that chain length distribution of side chains should make the peak lower, the broken rodlike star (eq 3) is a better model for the A3PQ samples than the fixed rodlike star.

The discrepancy between experimental and theoretical values for the highest $M_{\mathrm{w}}$ sample may be due to the finite chain flexibility. To consider this effect, the broken wormlike star model proposed by Huber and Burchard ${ }^{26}$ was utilized in which each arm connected with a 
universal joint as in the case of the broken rodlike star. In this model, the particle scattering function $P_{\mathrm{BW}}(q)$ is expressed as

$$
P_{\mathrm{BW}}(q)=\frac{2\left[\int_{0}^{L_{\mathrm{s}}}\left(L_{\mathrm{s}}-t\right) I\left(\lambda^{-1} q ; \lambda t\right) d t+\left(\int_{0}^{L_{\mathrm{s}}} I\left(\lambda^{-1} q ; \lambda t\right) d t\right)^{2}\right]}{3 L_{\mathrm{s}}^{2}}
$$

Here, $I\left(\lambda^{-1} q ; \lambda t\right)$ is the characteristic function of the wormlike chain, that is, the Fourier transform of the distribution function of the end-to-end distance of the wormlike chain of the contour length $t$ and of $\lambda^{-1}$. We utilized the Nakamura-Norisuye expression ${ }^{27-28}$ for the numerical calculation of $I\left(\lambda^{-1} q ; \lambda t\right)$. The theoretical $P_{\mathrm{BW}}(q)$ requires $L_{\mathrm{s}}$ and $\lambda^{-1}$ at given $q$. Assuming the formally determined values, that is, $h=0.19 \mathrm{~nm}$ and $\lambda^{-1}=43 \mathrm{~nm}$ for the corresponding linear PQX, we obtained theoretical blue dashed curves in Figure 4, for which the highest $M_{\mathrm{w}}$ sample reproduce the experimental values almost quantitatively. Furthermore, theoretical values for $P_{\mathrm{BW}}(q)$ become close to those for $P_{\mathrm{BR}}(q)$ with lowering $M_{\mathrm{w}}$ and they quantitatively explain the experimental data except for the lowest $M_{\mathrm{w}}$ sample, supporting the above mentioned suggestion for the bond angle between the two arbitrary arms. A further important point of the result is that chain length distribution of each arm may also be narrow while it is infeasible to be determined directly.

Molar Mass dependence of Dimensional and Hydrodynamic Properties. To confirm the above mentioned conformational properties, molar mass dependence of $\left\langle S^{2}\right\rangle_{\mathrm{z}}{ }^{1 / 2}$ was also analyzed in terms of the wormlike star models. The radius of gyration $\left\langle S^{2}\right\rangle_{\mathrm{BW}}$ for the wormlike star of which each arm is linked with a universal joint can be calculated by the Mansfield-Stockmayer scheme. $^{29}$ In the case of three-arm star, $\left\langle S^{2}\right\rangle_{\mathrm{BW}}$ is written as

$$
\left\langle S^{2}\right\rangle_{\mathrm{BW}}=\frac{7 L_{\mathrm{s}}}{18 \lambda}-\frac{5}{12 \lambda^{2}}+\frac{3-2 e^{-2 \lambda L_{\mathrm{s}}}}{12 \lambda^{3} L_{\mathrm{s}}}-\frac{1-e^{-2 \lambda L_{\mathrm{s}}}}{24 \lambda^{4} L_{\mathrm{s}}^{2}}
$$

The $g_{\mathrm{s}}$ values for the rigid rod $\left(\lambda L_{\mathrm{s}}<<1\right)$ and the random coil $\left(\lambda L_{\mathrm{s}}>>1\right)$ are calculated to be $1 / 3$ and 7/9, respectively. The theoretical solid curve in Figure 3 calculated from eq 9 with $h=0.19$ $\mathrm{nm}$ and $\lambda^{-1}=43 \mathrm{~nm}$ fairly reproduces the experimental data. Slight underestimation is most likely due to both the molar mass distribution and arm length distribution. The dashed curve in

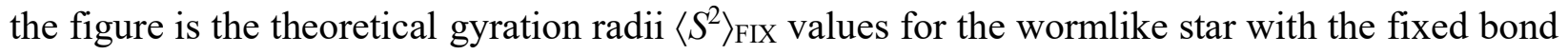
angle of $120^{\circ}$, which is also calculated by the same Mansfield-Stockmayer expression as follow

$$
\left\langle S^{2}\right\rangle_{\mathrm{FIX}}=\frac{7 L_{\mathrm{s}}}{18 \lambda}-\frac{1}{3 \lambda^{2}}+\frac{2-e^{-2 \lambda L_{\mathrm{S}}}}{12 \lambda^{3} L_{\mathrm{s}}}-\frac{1-e^{-4 \lambda L_{\mathrm{S}}}}{48 \lambda^{4} L_{\mathrm{s}}^{2}}
$$

The obtained theoretical values closely fit or slightly overestimate the experimental data, suggesting that the bond angle of the two arms is difficult to be estimated only from $\left\langle S^{2}\right\rangle_{z}$.

Molar mass dependence of the Flory viscosity factor $\Phi$ 


$$
\Phi=\frac{[\eta] M_{\mathrm{w}}}{\left(6\left\langle S^{2}\right\rangle_{\mathrm{z}}\right)^{3 / 2}}
$$

is illustrated in Figure 6. As in the case of the corresponding linear chain, $\Phi$ decreases with increasing $M_{\mathrm{w}}$ while the absolute values are systematically larger than those for the linear chains at the same $M_{\mathrm{w}}$. This is similar tendency for the formally investigated flexible star polymers. ${ }^{30-31}$ Since the current theories for $[\eta]$ of the wormlike star are only available for the fixed wormlike star, ${ }^{24}$ comparison of the experimental $[\eta]$ data with the theoretical values are not shown in this paper. It is however noted that analyses of $[\eta]$ will not give us the decisive information about the bond angle at the core because the relatively slight difference on $[\eta]$ from the bond angle restriction may be compensated by the chain thickness parameter. Indeed, if we choose $d=2.3$ $\mathrm{nm}$, the theoretical $[\eta]$ with the above mentioned $h$ and $\lambda^{-1}$ mostly fitted by the theory ${ }^{24}$ (not shown in the figure).

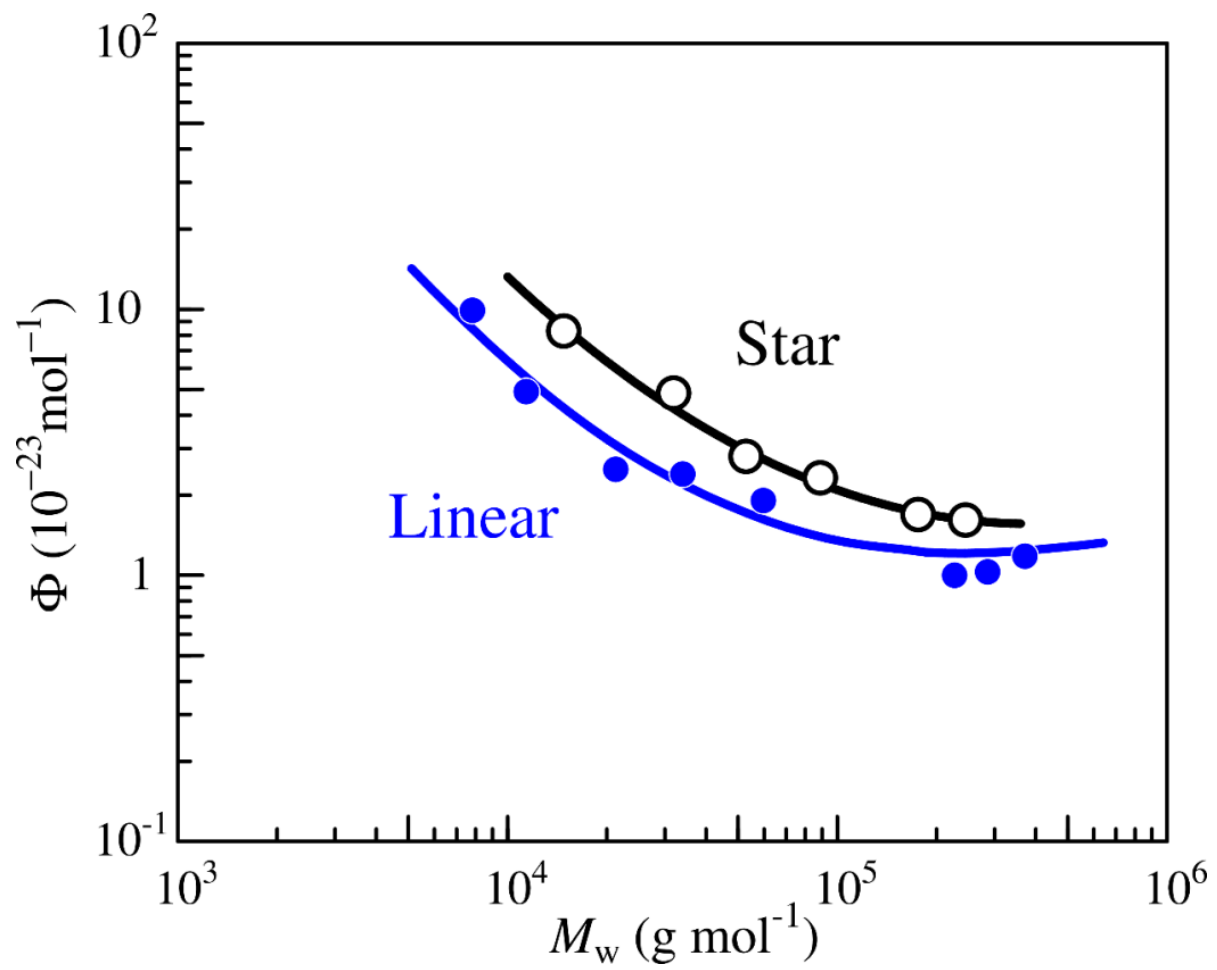

Figure 6. Double logarithmic plots of the Flory viscosity factor $\Phi$ vs $M_{\mathrm{w}}$ for A3PQ (unfilled circles) and PQX (filled circles) in $\mathrm{THF}$ at $25^{\circ} \mathrm{C}$.

\section{Conclusions}

We synthesized three-arm star poly[5,8-dimethyl-6,7-bis(propoxymethyl)quinoxaline-2,3diyl] (A3PQ) samples with various arm length by living polymerization using a new trifunctional palladium initiator. Solution properties characterized by the scattering function, the gyration radius, and the intrinsic viscosity were well explained by the broken wormlike star model, indicating that the arm length distribution is quite narrow and each arm connected with a rather 
flexible joint. In other words, A3PQ is a well-defined star polymer with uniform arm length and suitable as a good model as rigid star polymers.

\section{Supporting Information}

Additional experimental procedures, ${ }^{1} \mathrm{H}$ NMR spectra and Single-Crystal X-Ray Analysis of trimetallic initiator 2. This material is available free of charge via the Internet at http://pubs.acs.org.

\section{Acknowledgements}

The authors thank Prof. Takahiro Sato in Osaka University for valuable discussions and Dr. Noboru Ohta in SPring-8 for SAXS measurements. The synchrotron radiation SAXS experiments were performed at the BL40B2 in SPring-8 with the approval of the Japan Synchrotron Radiation Research Institute (JASRI) (Proposal Nos. 2015A1179, 2015B1100, and 2016B1088). The synchrotron radiation single-crystal X-ray experiments were performed at the BL40XU with the approval of the JASRI (Proposal Number 2016A1052, with Prof. Takuji Hatakeyama in Kwansei Gakuin University and Dr. Nobuhiro Yasuda in JASRI). This work was partly supported by JSPS KAKENHI Grant Nos. JP15H00994 (YN), JP16K05796 (YN), JP17H05368 (YN), JP25410130 (KT), and JP17K05884 (KT) and CREST, Japan Science and Technology Agency, "Establishment of Molecular Technology towards the Creation of New Function" Area (MS).

\section{References}

1. Hadjichristidis, N.; Pitsikalis, M.; Iatrou, H.; Driva, P.; Sakellariou, G.; Chatzichristidi, M. 6.03 - Polymers with Star-Related Structures: Synthesis, Properties, and Applications A2 Matyjaszewski, Krzysztof. In Polymer Science: A Comprehensive Reference, Möller, M., Ed. Elsevier: Amsterdam, 2012; pp 29-111.

2. Nakamura, Y.; Norisuye, T. 2.02 - Polymer Properties in Solutions. In Polymer Science: A Comprehensive Reference, Editors-in-Chief: Krzysztof, M.; Martin, M., Eds. Elsevier: Amsterdam, 2012; pp 5-32.

3. Yamakawa, H.; Yoshizaki, T. Helical Wormlike Chains in Polymer Solutions, 2nd ed. Springer: Berlin, Germany, 2016.

4. Oka, M.; Nakajima, A. Synthesis and Characterization of Three-branched Star-like Poly(g-benzyl-l-glutamate). Polym. Bull. 1984, 11, 447-451.

5. Goodson, S. H.; Novak, B. M. Synthesis and Characterization of Wormlike Three-arm Poly(n-hexyl isocyanate) Star Polymers. Macromolecules 2001, 34, 3849-3855.

6. Kikuchi, M.; Takahara, A.; Kawaguchi, S. Dimensional Characterizations from Rod Stars to Brushes of Polymers with a Low Degree of Polymerization. Macromolecules 2016.

7. Sanada, Y.; Shiomi, T.; Okobira, T.; Tan, M.; Nishikawa, M.; Akiba, I.; Takakura, Y.; Sakurai, K. Polypod-Shaped DNAs: Small-Angle X-ray Scattering and Immunostimulatory Activity. Langmuir 2016, 32, 3760-5.

8. Miyabe, T.; Iida, H.; Banno, M.; Yamaguchi, T.; Yashima, E. Synthesis and Visualization of a Core Cross-Linked Star Polymer Carrying Optically Active Rigid-Rod Helical Polyisocyanide Arms and Its Chiral Recognition Ability. Macromolecules 2011, 44, 8687-8692.

9. Knoop, R. J. I.; de Geus, M.; Habraken, G. J. M.; Koning, C. E.; Menzel, H.; Heise, A. Stimuli Responsive Peptide Conjugated Polymer Nanoparticles. Macromolecules 2010, 43, 4126-4132. 
10. Skey, J.; Willcock, H.; Lammens, M.; Du Prez, F.; O'Reilly, R. K. Synthesis and SelfAssembly of Amphiphilic Chiral Poly(amino acid) Star Polymers. Macromolecules 2010, 43, 5949-5955.

11. Siriwardane, D. A.; Kulikov, O.; Reuther, J. F.; Novak, B. M. Rigid, Helical Arm Stars through Living Nickel Polymerization of Carbodiimides. Macromolecules 2017, 50, 832-840.

12. Ida, D. Dilute Solution Properties of Semiflexible Star and Ring Polymers. Polym. J. 2014, 46, 399-404.

13. Terao, K.; Mizuno, K.; Bachinger, H. P. Conformational change from rigid rod to star: a triple-helical peptide with a linker domain at the C-terminal end. J. Phys. Chem. B. 2015, 119, 3714-9.

14. Nagata, Y.; Hasegawa, H.; Terao, K.; Suginome, M. Main-Chain Stiffness and Helical Conformation of a Poly(quinoxaline-2,3-diyl) in Solution. Macromolecules 2015, 48, 7983-7989.

15. Yamamoto, T.; Suginome, M. Helical Poly(quinoxaline-2,3-diyl)s Bearing Metal-binding Sites as Polymer-based Chiral Ligands for Asymmetric Catalysis. Angew. Chem. Int. Ed. 2009, 48, 539-42.

16. Yamada, T.; Nagata, Y.; Suginome, M. Non-hydrogen-bonding-based, solvent-dependent helix inversion between pure P-helix and pure M-helix in poly(quinoxaline-2,3-diyl)s bearing chiral side chains. Chem. Commun. 2010, 46, 4914-6.

17. Nagata, Y.; Yamada, T.; Adachi, T.; Akai, Y.; Yamamoto, T.; Suginome, M. Solventdependent Switch of Helical Main-chain Chirality in Sergeants-and-soldiers-type Poly(quinoxaline-2,3-diyl)s: Effect of the Position and Structures of the "Sergeant" Chiral Units on the Screw-sense Induction. J. Am. Chem. Soc. 2013, 135, 10104-13.

18. Kotha, S.; Kashinath, D.; Lahiri, K.; Sunoj, Raghavan B. Synthesis of C3-Symmetric Nano-Sized Polyaromatic Compounds by Trimerization and Suzuki-Miyaura Cross-Coupling Reactions. Eur. J. Org. Chem. 2004, 2004, 4003-4013.

19. Ito, Y.; Ohnishi, A.; Ohsaki, H.; Murakami, M. A Preparative Method foroDiisocyanoarenes. Synthesis 1988, 1988, 714-715.

20. Ito, Y.; Ihara, E.; Uesaka, T.; Murakami, M. Synthesis of Novel Thermotropic Liquid Crystalline Poly(2,3-quinoxalines). Macromolecules 1992, 25, 6711-6713.

21. Berry, G. C. Thermodynamic and Conformational Properties of Polystyrene. I. LightScattering Studies on Dilute Solutions of Linear Polystyrenes. J. Chem. Phys. 1966, 44, 45504564.

22. Grubisic, Z.; Rempp, P.; Benoit, H. A Universal Calibration for Gel Permeation Chromatography. J. Polym. Sci. Pol. Lett. 1967, 5, 753-759.

23. Pasch, H. 2.03 - Chromatography A2 - Matyjaszewski, Krzysztof. In Polymer Science: A Comprehensive Reference, Möller, M., Ed. Elsevier: Amsterdam, 2012; pp 33-64.

24. Ida, D.; Nakamura, Y.; Yoshizaki, T. Intrinsic Viscosity of Wormlike Regular Three-arm Stars. Polym. J. 2008, 40, 256-267.

25. Irurzun, I. M. Hydrodynamic Properties of Regular Star-branched Polymer in Dilute Solution. J. Polym. Sci., Part. B: Polym. Phys. 1997, 35, 563-567.

26. Huber, K.; Burchard, W. Scattering Behavior of Wormlike Star Macromolecules. Macromolecules 1989, 22, 3332-3336.

27. Nakamura, Y.; Norisuye, T. Brush-Like Polymers. In Soft Matter Characterization, Borsali, R.; Pecora, R., Eds. Springer Netherlands: 2008; pp 235-286.

28. Nakamura, Y.; Norisuye, T. Scattering Function for Wormlike Chains with Finite Thickness. J. Polym. Sci., Part. B: Polym. Phys. 2004, 42, 1398-1407. 
29. Mansfield, M. L.; Stockmayer, W. H. Unperturbed Dimensions of Wormlike Stars. Macromolecules 1980, 13, 1713-1715.

30. Roovers, J.; Toporowski, P. M. Hydrodynamic Studies on Model Branched Polystyrenes. J. Polym. Sci., Part. B: Polym. Phys. 1980, 18, 1907-1917.

31. Okumoto, M.; Terao, K.; Nakamura, Y.; Norisuye, T.; Teramoto, A. Excluded-volume effects in star polymer solutions: Four-arm star polystyrene in cyclohexane near the Theta temperature. Macromolecules 1997, 30, 7493-7499. 
For Table of Contents Use Only

Synthesis and Solution Properties of a Rigid Helical Star Polymer: Three-arm Star Poly(quinoxaline-2,3-diyl)

Hirokazu Hasegawa, Yuuya Nagata, Ken Terao, and Michinori Suginome

Core-first Method

\section{Living Polymerlization}

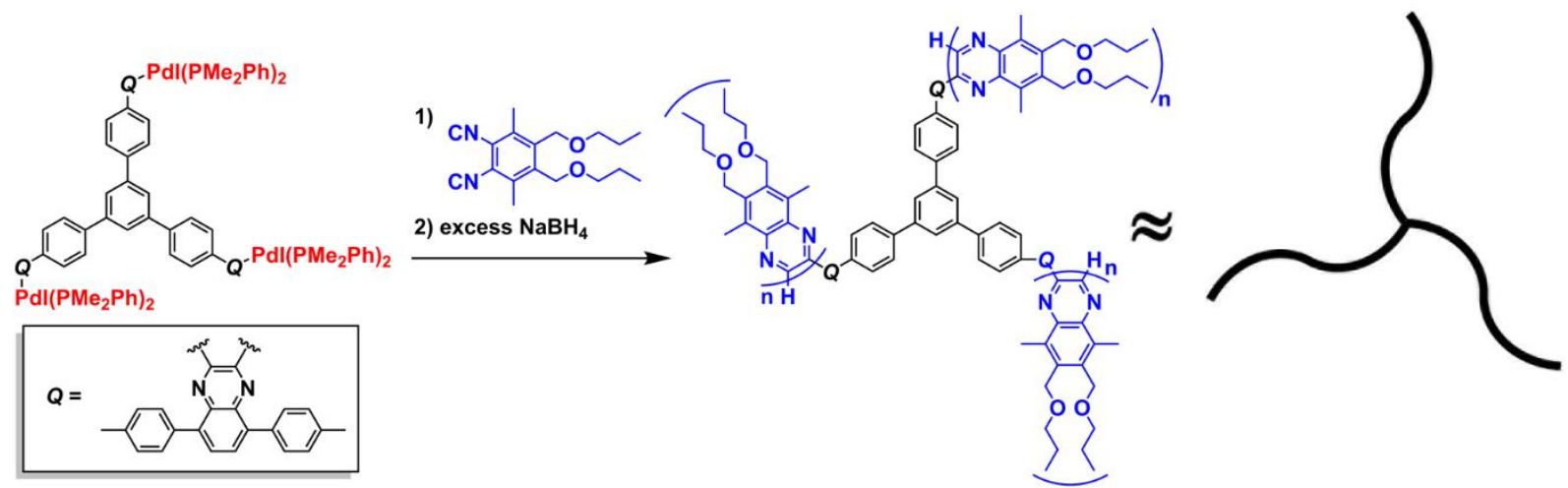

Solution Properties Reveals Regular Wormlike Three-Star 